be available for use. The speaker had been trying to establish whether or not drug-resistant patients were contacts of foreign troops but had not found any evidence to support the suggestion.

Maj. C. S. Nicol said that he was very interested to hear Lt.-col. Campbell's account of his experience in Italy, in the course of which he had mentioned a case of anuria which in spite of ureteric catheterization had terminated fatally. There had been at least one similar case described in the United States in which it was found at necropsy that there was renal damage with small haemorrhages round the tubules as well as the mechanical block of the ureters. Gp.-capt. McElligott had made a point of the importance of waiting for ten days before starting sulphonamide therapy in order to raise body immunity. This was a matter of which the speaker had had no experience; but there were other ways of stimulating immunity. Even if mechanical fever therapy was not available there was the possibility of raising immunity by means of intravenous anti-typhoid-paratyphoid vaccine. In reply to Gp.-capt. McElligott's comments on Group $\mathbf{C}$ cases almost, but not quite all, cases in the group had had a second course of sulphonamide. The figures given in the recent paper by Gp.-capt McElligott and Sqn.-ldr. Jefferiss, when compared with the figures given in the paper under discussion, would be found to be very similar if only gonococcus-fast cases in Group C were counted as failures. Sqn.-ldr. Jefferiss had emphasized the importance of inspecting the all-night urine, a point with which the speaker was in complete agreement. For workers in civil olinics one of the difficulties of assessing clinical cure was that they were not able to see the patient's all-night urine; this was not so in the Forces as the patient was in hospital during treatment. Cases with a purulent urethral discharge and a crystal clear all-night urine did occur, however. As regards the question about the shortest number of days during which sulphathiazole should be given, they had had experience only of the 3-day course, and the point which Sqn.-ldr. Jefferiss had made concerning the efficiency of a 2-day course had not been investigated. The speaker expressed great interest in Gen. Biggam's remarks regarding the dangers of using T.A.B: vaccine intravenously and would like to know whether the fatality mentioned was due to heat stroke or to liver damage ; if the latter, had an aetiological factor been suggested either of the nature of an infective agent or of a dietetic deficiency? He had not seen fatalities or any patients seriously ill after the employment of T.A.B. vaccine. The very small doses used-small compared with the size of the dose which Dr. Hanschell mentioned, for instance-were sufficient to obtain the desired fever response. Two divided doses of 25 million organisms were used and it was found that these gave a satisfactory temperature elevation without any danger to the patient.

Sqn.-ldr. Morton had raised a point with regard to the question of desensitization. The cases they had had to desensitize were of patients who in the ordinary way would not be able to receive a further course of sulphathiazole because it had already been administered to them and a pyrexial reaction or a generalized rash had developed. Only these few cases had been desensitized as described. Sulphathiazole given initially in small doses and gradually increased to full dosage was better than no sulphathiazole at all.

Maj. D. J. Williams added that he did not think the 2-day course of 5 grammes per day was successful, in that 20 per cent of patients who had been treated by unit medical officers by that method had had to be sent to hospital for further treatment because they showed positive smears.

\title{
A JOINT VENEREAL DISEASES SERVICE FOR THREE RURAL OR URBAN DISTRICTS
}

\author{
By A. N. P. MILNER, M.B., B.Ch. \\ Hon. Dermatologist, City Lodge Hospital, Cardiff
}

\section{GENERAL PLAN}

This scheme (see coloured diagram) is drawn up on the assumption that the service may be directed from a venereal diseases service centre situated in one district, which shall be known as the central district, and that it may operate in and around three separate district V.D. clinics.

The service is organized in three sectors: (1) the service clinical section, in charge of the specialist officer, (2) the service pathology section, in charge of the V.D. medical pathologist and (3) the service almoner's section, in charge of the lady almoner. These sections will be administered from three adjacent central offices in the district.

The central direction of the service will be undertaken by the three officers in council with the medical officers of health of the three districts, or the administrative medical officer whom they may jointly appoint to represent them, and a representative of the Ministry of Health. 
DIAGRAM OF THREE DISTRIC $\frac{\bar{m}}{\mathrm{a}} \mathrm{I}$ V.D. ORGANISATION

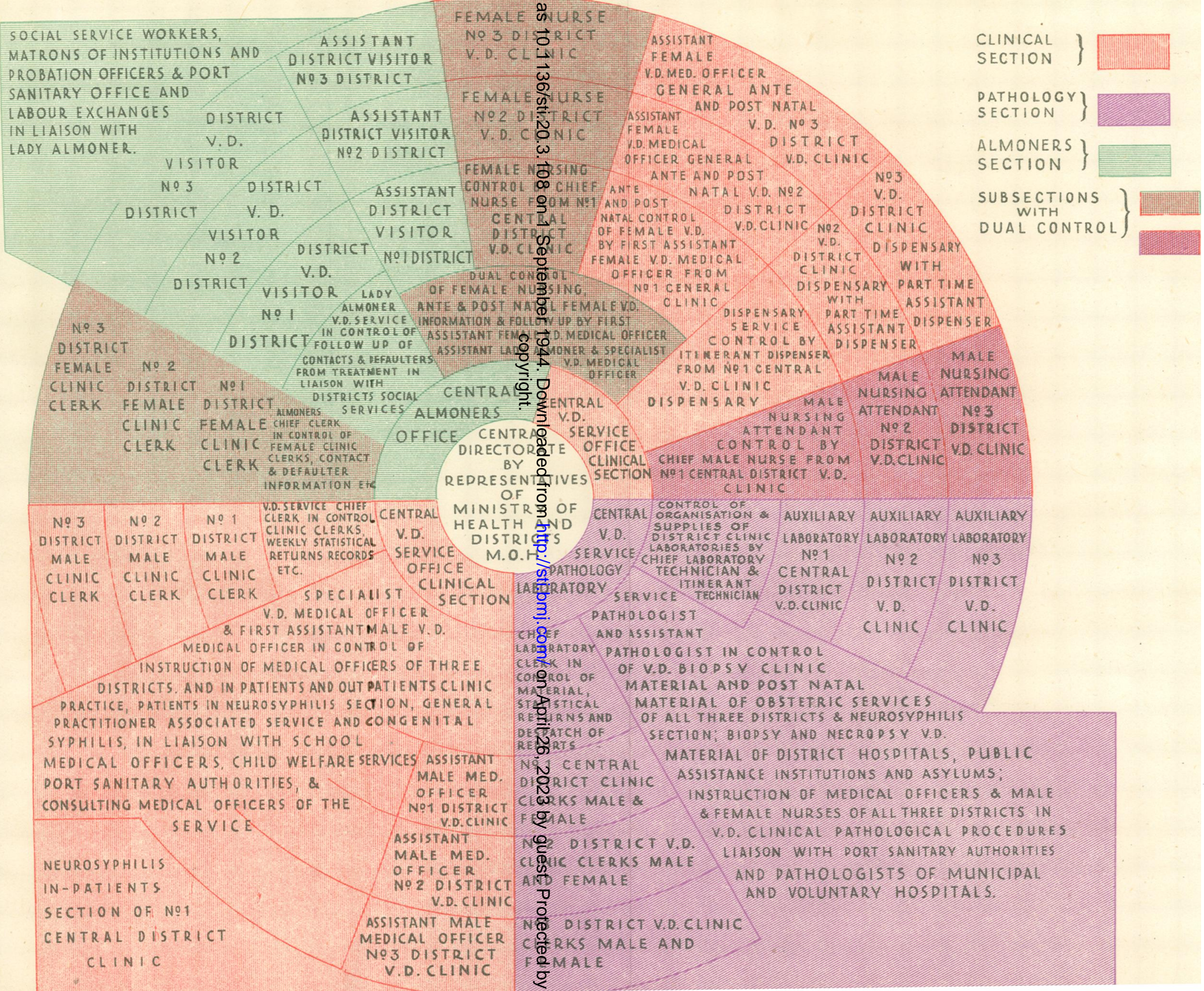


The general executive council of the service will consist of the officers in charge of sectors and their chief assistants who will then direct their sections in accordance with the recommendation of the council.

The specialist venereal diseases officer will be assisted by the first assistant male venereal diseases medical officer and the first assistant female venereal diseases medical officer.

The pathologist will be assisted by the assistant pathologist and the lady almoner by the assistant lady almoner.

The service clinical section will control the following activities and staff.

(1) Clinical practice on all V.D. in-patients and out-patients of the three district clinics

and the central neurosyphilis section.

(2) Direction of treatment routines to be carried out by general practitioners employed by the service.

(3) Organization of district clinics.

(4) V.D. male attendants of the service.

(5) V.D. clinic equipment.

(6) The service dispenser and the district clinic dispensaries and supplies.

(7) Female V:D. nurses of the service in liaison with the assistant lady almoner.

(8). Instruction of staff in charge of V.D. clinics, antenatal and postnatal service medical

officers, obstetric service medical officers and general practitioners employed by the service.

(9) District clinic clerical service.

(10) Direction of treatment of V.D. in-patients of the Public Assistance county institutions and county hospitals.

(11) V.D. prophylactic centres and measures.

(12) Lectures to the general public on V.D.

(13) Clinical research work conducted by the service.

The pathological section will control the following branches of the work.

(1) Pathological investigations on all V.D. patients of the three districts.

(2) Auxiliary laboratories of the district clinics organization, supplies and traffic with the central laboratory.

(3) Clerical duties, in so far as they concern pathology, of the district clinic clerks.

(4) Instruction of district clinic male attendants in simple laboratory procedures used at auxiliary laboratories.

(5) Instruction in the pathology of venereal diseases of the V.D. service clinic staff, of general practitioners employed by the service, of antenatal and postnatal medical officers and of obstetrical medical officers.

(6) Biopsy material from all V.D. patients.

(7) Investigation of placentas and umbilical cords of all children born of syphilitic mothers.

(8) Pathology laboratory equipment and supplies.

(9) Clinical pathology research work conducted by the service.

(10) Pathology section staff.

(11) Necropsy material of all V.D. patients who die in county hospitals or other institutions or who are brought to the coroner's mortuary, if permission can be obtained.

The lady almoner's section will control branches of the work as follows.

(1) Tracing and bringing to treatment of contacts, male and female, notified under 33B, working in liaison with the district medical officers of health.

(2) Continued observation of antenatal and postnatal mothers, in liaison with obstetrical antenatal and postnatal services and county institutions (assistant almoner).

(3) Detection of patients who default from treatment, in liaison with the district medical officers of health.

(4) Control of the movements of the floating V.D. population, in liaison with the labour exchanges of the three districts and the lady almoners of other districts.

(5) Maintenance of contact with children who are infected with V.D., in liaison with child welfare associations and school nursing services (assistant almoner).

(6) Supervision, discipline and nursing technique of the female V.D. district clinics, in liaison with the first assistant V.D. lady medical officer through the assistant lady almoner.

(7) District V.D. visitors of all three districts.

(8) Clerical duties of district clerks in this section.

(9) Instruction of almoner district clerks and district V.D. visitors, welfare workers and school nurses in almoner service duties.

(10) Liaison with district probation officers.

(11) Finding of occupational posts for idle and promiscuous females who are infected with V.D., while they are under treatment and after-they are cured.

(12) Finding of homes for illegitimate children born of syphilitic mothers, in liaison with adoption societies, obstetrical and postnatal services and county institutions. 
Itinerant medical staff

\section{MEDICAL STAFF}

The members will work together as a team and serve at all three district V.D. clinics.

(1) Specialist V.D. medical officer.

(2) First assistant male medical officer.

(3) First assistant female V.D. medical officer.

(4) One trained laboratory technician (whole-time) who could be employed at the central laboratory when not on duty at district clinics.

(5) One trained V.D. male attendant, who when not on duty at other district clinics could assist the senior male attendant of the service at No. 1 central district clinic, and take charge during the latter's absence on supervisory visits to other district clinics.

(6) One female trained V.D. nurse, who when not on duty at other district clinics could assist the senior female V.D. nurse of the service at No. 1 central district clinic, and take charge during the latter's absence on supervisory visits to other clinics.

(7) One dispenser.

\section{Stationary medical staff}

The members will be employed at district clinics.

(1) Two part-time* male medical officers, one for No. 2 and one for No. 3 district clinic. These will assist at four clinic sessions at their respective district clinics. They might be chosen from general medical practitioners, school or antenatal medical officers.

(2) Two part-time* female medical officers, one for No. 2 and one for No. 3 district clinic. These will assist at four clinic sessions at their respective district clinics. They might be chosen from general medical practitioners, obstetrical, antenatal or school medical officers.

(3) One senior male attendant of the service (whole-time), who will be stationed at No. 1 district clinic but will make weekly supervisory visits to the other two district clinics and will be in charge of the other two assistant male attendants.

(4) Two assistant male attendants (whole-time), one at No. 2 and one at No. 3 clinic, stationed at and in charge of their respective district clinics.

(5) One senior female nurse of the service (whole-time), who will be stationed at No. 1 central clinic but will make weekly supervisory visits to each of the other district clinics.

(6) Two assistant female nurses (whole-time), who will be stationed one at No. 2 and one at No. 3 district clinic. They will be in charge of the female and antenatal sections of their respective clinics.

(7) Three pupil male attendants (part-time), one at each clinic.

(8) Three pupil female attendants (part-time), one at each clinic.

(9) Three part-time dispensers, one at each clinic.

*Note. It may be necessary later on to make the part-time assistant male and female medical officers whole-time officers, and to let them share in lecturing to the general public and to medical officers and take charge of their respective clinics.

\section{Pathological service staff}

(1) One whole-time pathologist, trained in the clinical pathological techniques required for the investigation of all varieties of venereal disease.

(2) One part-time assistant pathologist, to be trained in V.D. clinical pathology and to become whole-time if the amount of work should require it.

(3) One fully trained laboratory senior technician.

(4) One trained laboratory junior technician, who will work in an itinerant capacity during V.D. sessions at all district clinics and assist at the central laboratory when not employed at the clinics.

(5) Two laboratory assistants, who will be trained as V.D. laboratory technicians.

(6) One typist clerk (female).

(7) One senior reception office attendant (female).

(8) One junior reception office attendant (female).

\section{Central V.D. office staff}

(1) Special venereologist medical officer.

(2) First assistant medical officer (male).

(3) First assistant V.D. medical officer (female).

(4) One chief service clerk (male).

(5) One typist secretary (female).

(6) One office assistant (female).

(7) Three male clinic clerks, one for each district clinic.

\section{Almoner's staff}

(1) One whole-time lady almoner, trained.

(2) One whole-time assistant lady almoner. Note. One of the two lady almoners should have been trained up to senior hospital staff nurse or hospital sister standard for the purpose of nursing control. 


\section{A JOINT VENEREAL DISEASES SERVIĆE}

(3) One almoner's chief clerk (female), to 'be stationed at central almoner's office.

(4) Two typist clerks (female).

(5) One receptionist office assistant (female).

(6) Three clinic clerks (female), stationed one at each of the district clinics, who could also be employed as part-time district visitors.

(7) Six whole-time district visitors (female), to be stationed two in each district.

(8) Three part-time district visitors (female), to be stationed one in each district. They could be employed at school or antenatal clinics or as ordinary health visitors when they are not working on V.D. cases which require subsequent observation or on those which come under Regulation 33B.

\section{CLINICAL SERVICE}

Clinical practice should be directed from three district clinics situated at a site in each district of easy access from all sections of the district, and adjacent to some county institution which could provide beds for V.D. patients.

Each district V.D. clinic should be constructed as shown in the plan on page 114. It is most important that the structures should be built in relation to the points of the compass, as shown in the plan, so as to bring the maximum of light from the south and west into the treatment rooms and consulting rooms.

Each district clinic will provide facilities as follows. (1) Two general adult clinics and two clinics for antenatal and postnatal V.D, patients and for children suffering from venereal diseases. Male and female clinics should be conducted concurrently in completely separate sections of each district clinic (see plan). Later, it may become necessary to institute a third general adult clinic at each. (2) Daily local treatment of male and female patients by the respective attendants at three morning and three evening sessions each week, and for prophylactic measures under the direction of the district clinic medical officers. (3) Facilities whereby general medical practitioners of the district who are employed by the V.D. service may have biopsy material examined at the clinic auxiliary laboratory in order to promote early diagnosis.

The service should, in addition, provide a neurosyphilis section, to be situated in one of the county institutions adjacent to the central No. 1 district clinic and in the same building as the ordinary V.D. beds attached to the central clinic. This section should be served by fully trained male and female nurses, and should provide facilities for fever therapy for neurosyphilis and resistant gonorrhoea by malaria and the Kettering hypertherm cabinet.

Each district clinic should be staffed as follows.

(1) One male attendant (whole-time) plus one pupil male attendant (part-time).

(2) One female nurse (whole-time) plus one pupil nurse (part-time).

(3) One male clinic clerk (whole-time).

(4) One female clinic clerk (whole-time).

(5) One engineer to be in charge of the central heating, sterilizing and electrical arrangements (whole-time).

(6) Adequate staff of cleaners (whole-time).

(7) One male assistant V.D. medical officer (whole-time or part-time, according to district requirements).

(8) One female assistant V.D. medical officer (whole-time or part-time according to district requirements). This assistant should also be trained in antenatal, postnatal and obstetrical work, and have some knowlejge of diseases of children.

(9) One dispenser (part-time).

Note. It would be better if the medical officers (7) and (8) could be employed as wholetime officers and take their share of service duties in their respective districts.

The clinic will be under the control of the male and female nurses, directed by the male and female medical officers.

The ward beds attached to the clinic will be attended by the male and female clinic nurses under the direction of the clinic medical officers, who will make daily rounds of the beds in their charge.

The specialist V.D. officer will preside over one general adult clinic and one combined antenatal, postnatal and children's clinic in each district each week ; his work will be to examine and prescribe for new cases and resistant or otherwise abnormal cases, to assess the results of final tests for cure and to supervise the final discharge of patients from treatment. Each clinic which he attends will be 
conducted as a teaching clinic at which practical instruction will be given to pupil medical officers of the service. Before or after each clinic session he will visit the V.D. beds attached to the clinic.

During the inauguration of the service, and from time to time throughout his service, he will arrange to give courses of lectures to the following assistants in each district.

(1) General practitioners and medical officers of the obstetric, antenatal, postnatal,

child welfare and school medical associated services of each district,

(2) Lecturers to the general public on V.D., to instructors of Youth Groups, and to

those training to be school teachers and children's nurses.

(3) Male and female V.D. nursing clinic staff and pupil nurses.

From time to time he will summon and preside at meetings of the service executive councils. He will attend meetings of the central directorate when called upon to do so.

The first assistant male V.D. medical officer will be under the direction of the specialist V.D. officer and will cooperate with him and with the first assistant female V.D. medical officer in every way. His main duties will be as follows.

(1) To assist the specialist officer at one general adult clinic and one combined antenatal and postnatal V.D. and children's clinic at No. 1 central district clinic each week and to accompany him on his weekly ward visits to the V.D. beds and neurosyphilis section attached to the central district clinic.

(2) To deputize for the specialist officer at one general adult clinic and at one visit to the V.D. wards once each week in each district and at the clinical section of the central administrative service office on two mornings of each week.

(3) To give practical instruction to the male staff of the central district clinic once each week in liaison with the first assistant female V.D. officer, the assistant lady almoner and the chief clerks of the almoner and clinical sections.

(4) To visit the V.D. wards attached to the No. 1 central clinic and those of the neurosyphilis section, of which he will have joint charge with the first assistant female V.D. officer, daily.

(5) To lecture to the male general public and assist with lectures to medical officers from time to time under the direction of the specialist V.D. officer.

(6) To-administer the congenital syphilis section and the V.D. prophylactic arrangements of the V.D. service under the direction of the specialist V.D. officer and in liaison with the first assistant female V.D. officer, and with the school medical officers', child welfare officers and general practitioners of all three districts.

(7) To take charge of a mobile unit twice weekly for service where necessary in the three districts with the assistance of the itinerant male attendant, the itinerant female nurse and the itinerant laboratory technician.

The first assistant female V.D. medical officer will work under the direction of the specialist V.D. officer and will cooperate with him and with the first assistant male V.D. officer in every way. Her main duties will be as follows.

(1) To deputize for the specialist V.D. officer at one combined antenatal, postnatal and children's clinic, at one ward visit of the female and children's V.D. beds once each week in each district-and at the clinical section of the central administrative service office on two mornings each week.

(2) To assist the specialist V.D. officer at one general ádult clinic and one combined antenatal, postnatal and children's clinic at No. 1 central district clinic and to accompany him on his weekly ward visit to the V.D. beds and neurosyphilis section attached to the central clinic.

(3) To take joint charge with the first assistant male medical officer of the V.D. beds attached to No. 1 central clinic and those of the neurosyphilis section, which she will visit daily.

(4) To administer the antenatal, postnatal and obstetrical section of the V.D. service under the direction of the specialist V.D. Officer and in liaison with the associated medical officers, nursing staff and district visitors of all three districts.

(5) To give practical instruction to the female staff of No. 1 central district clinic once each week, in liaison with the first assistant male medical officer, the assistant lady almoner and the chief clerks of the almoner and clinical sections.

(6) To lecture on V.D. nursing and control to the female staffs of the antenatal, postnatal and obstetrical services of each district, at regular intervals.

(7) To lecture to the female general public on V.D. and assist with the lectures to medical officers from time to time under the direction of the specialist V.D. officer.

(8) To take charge of a mobile unit for female and child patients, twice weekly, for service where necessary in all three districts, with the assistance of the itinerant laboratory assistant and the itinerant female nurse. 


\section{CLINIC CONSTRUCTION}

Clinics should be constructed according to the rough plan on page 114, which can be modified to meet architects' requirements.

The dispensary should have two skylights and shaft ventilation.

The dividing wall between male and female waiting rooms should be fairly thick and sound-proof. The dividing partitions of the cubicles in the treatment rooms should be substantial: Treatment cubicles in both treatment rooms and irrigation cubicles in the male treatment room should be closed by runner white curtains over entrances.

\section{General}

Heating.-The heating plant should provide central heating and hot and cold water for the whole clinic, as well as steam for three box steam sterilizers (one in each of rooms 18 . 21 and 24) and steam for drying cupboards in linen room and in staff and medical officers' cloakrooms.

Central sterilizing plant.-This should be capable of sterilizing large dressing drums. (If a scabies treatment centre is stationed near the clinic, which would be a distinct advantage, this plant could be used to sterilize the clothes and bed linen of the scabies patients.)

Windows.-These should be of the casement type, and large.

Lighting.- This should be plentiful and from ceiling diffused electric lights. Treatment rooms 21 and 24 should have anglepoise electric lights attached to one wall of each cubicle. Rooms 19, 20, 22 and 23 should be provided with anglepoise lamps lit by daylight bulbs of 100 candle-power.

Plumbing.-Rooms 17, 18, 19, 20, 22 and 23 should be fitted with one hot and cold water lavatory basin. Rooms 2, 21, 24 and 29 should be fitted with two hot and cold water lavatory basins. Rooms 21 and 24 should be fitted with one hot and cold water sluicing sink. Room 18 should be fitted with two hot and cold water lavatory sinks. Two flushing water closets should be provided in each of rooms 2 and 29 . Two flushing water closets and two hot and cold water lavatory basins should be provided in waiting room lavatories, 13 and $25 \mathrm{~A}$, and in treatment room lavatories $21 \mathrm{E}$ and $24 \mathrm{E}$. Two flushing water closets should be provided for laboratory lavatory $18 \mathrm{~A}$.

Electric power.-This should be provided as follows.

(1) In rooms 21 and 24 to serve two electric sterilizers.

(2) In rooms $19,20,22$ and 23 to serve one electric sterilizer.

(3) As lighting wall plugs in each cubicle of treatment rooms 21 and 24 for use with electric instruments from light transformers.

(4) In room 18 to serve one electric incubator, one electric centrifuge, and one electric - refrigerator.

(5) In rooms $3,6,12,17,18,19,20,21,22,23,24,25$ and 28 to serve electric wall radiators to replace central heating on cold days in summer and should the central heating plant fail.

(6) In room 31 to serve a small electric cooker and a small electric refrigerator.

(7) In room 17 to serve a small electric refrigerator.

Coal gas.-This should be supplied (1) to the laboratory (room 18), for gas incubator, water bath and Bunsen burners, and (2) to the dispensary (room 17).

\section{Furniture}

Consulting rooms 19 and 22 and reception rooms 20 and 23.-Desk, desk chair, patient's chair, consulting-room couch, electric sterilizer, hot and cold water lavatory basin, instrument cupboard, anglepoise lamp fitted with daylight bulb.

Female treatment room, 21.-Three partitions separating cubicles each fitted with anglepoise lamp attached to partition and having a daylight bulb. One porcelain-topped table. Five straight chairs. Two hot and cold water lavatory basins. One hot and cold water sluice sink. One box steam sterilizer. Two electric sterilizers. Three adjustable metal. urological chairs painted white enamel. One instrument cupboard.

Male treatment room, 24.-Two partitions separating cubicles, each fitted with anglepoise lamps with daylight bulbs. Two adjustable metal urological chairs painted white enamel. One porcelain-topped table. Five straight chairs. Two hot and cold water lavatory basins. One hot and cold water sluice sink. One box steam sterilizer. Two electric sterilizers. Six irrigating cubicles with automatic water flushing metal receptacles. One instrument cupboard.

Clinic auxiliary laboratory, room 18.-One electric refrigerator. One electric centrifuge. One gas or electric incubator. One box steam sterilizer. Working bench fitted with two laboratory sinks. Three gas taps and three Bunsen burners. Four cupboards fitted with glass door and shelves fitted above bench. One hot and cold water lavatory basin. One desk, filing cupboards, store cupboards, tables and chairs. One water distilling set. 
THE BRITISH JOURNAL OF VENEREAL DISEASES
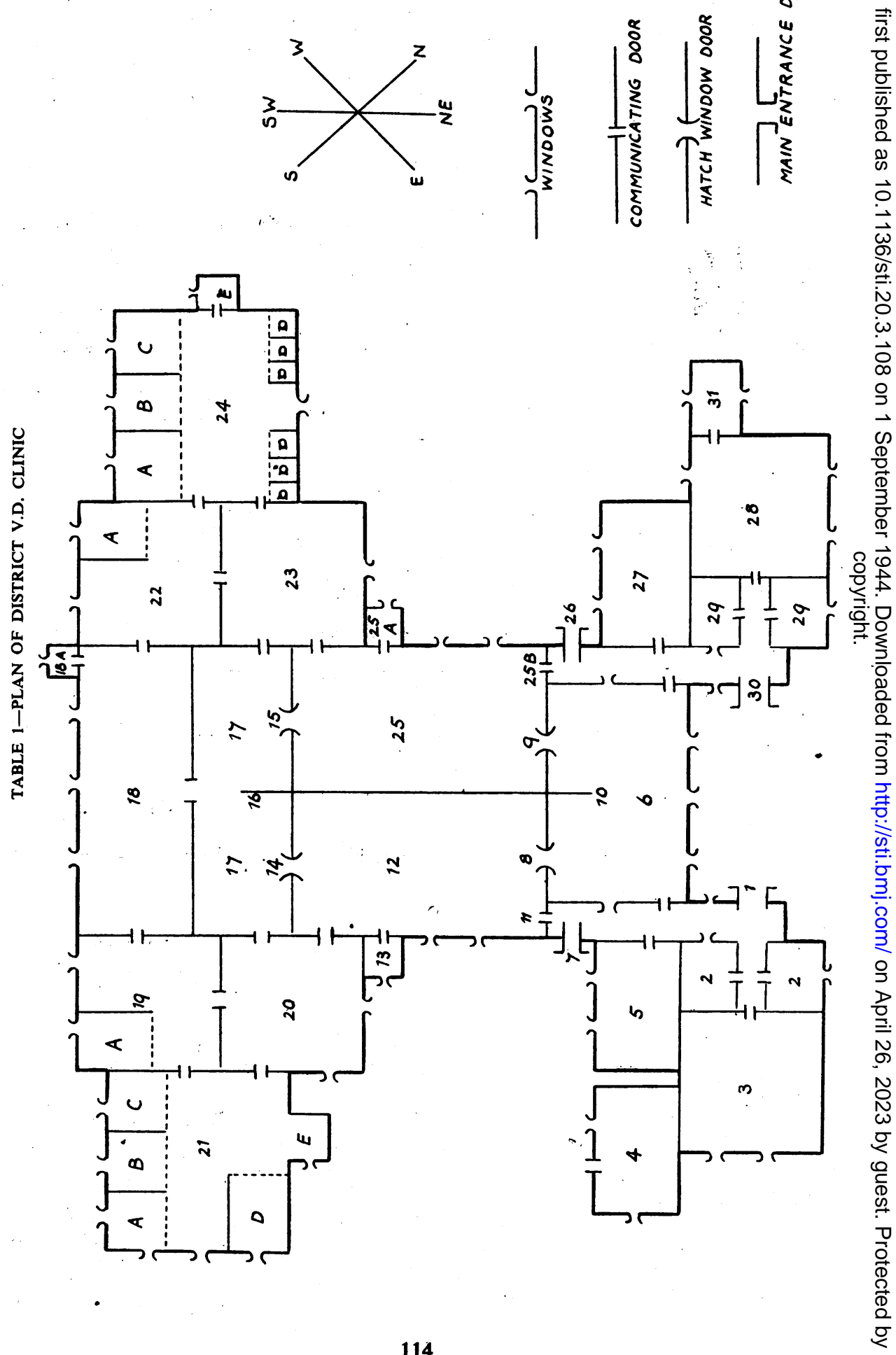

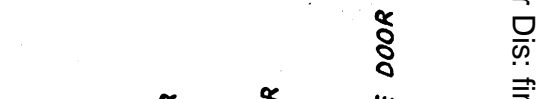


Medical officers' entrance

Medical officers' cloakroom and lavatories, $10^{\prime} \times 5^{\prime} \times 10^{\prime}$

Medical officers' sitting room, $10^{\prime} \times 10^{\prime} \times 10^{\prime}$

Central heating and sterilizing plant, $8^{\prime} \times 6^{\prime} \times 10^{\prime}$

Linen room, $6^{\prime} \times 10^{\prime} \times 10^{\prime}$

Clinic clerks' office, $15^{\prime} \times 12 t^{\prime} \times 15^{\prime}$

Female patients' entrance

Hatch-window communicating

Partich communicating with male waiting room

Partition $5^{\prime} \times 10^{\prime}$ dividing communication cubicles of clerks' office

Entrance to female waiting room

Female patients' waiting room, 17 $\xi^{\prime} \dot{\times} 10^{\prime} \times 15^{\prime}$

Waiting room lavatory

Hatch-door communicating with female waiting room

Hatch-door communicating with male waiting room

Partition $4^{\prime} \times 10^{\prime}$ separating communication cubicles of dispensary

Dispensary, $20^{\prime} \times 8^{\prime} \times 15^{\prime}$. This should have a skylight and ventilator

Laboratory, $20^{\prime} \times 8^{\prime} \times 15^{\prime}$. $18 \mathrm{~A}$, laboratory lavatory

Female consulting room, $10^{\prime} \times 10^{\prime} \times 10^{\prime}$. $19 \mathrm{~A}$, examination cubicle

Female reception room, $10^{\prime} \times 10^{\prime} \times 10^{\prime}$

Female treatment room, $13^{\prime} \times 13^{\prime} \times 10^{\prime}$. 21A, B, C, D, treatment cubicles. 21E, treatment room lavatory

Male consulting room, $10^{\prime} \times 10^{\prime} \times 10^{\prime} . \quad 22 \mathrm{~A}$, oxamination cubicle

Male reception room, $10^{\prime} \times 10^{\prime} \times 10^{\prime} \times 13^{\prime} \times 24 \mathrm{~A}, \mathrm{~B}, \mathrm{C}$, treatment room cubicles, $4^{\prime} \times 5^{\prime} \times 10^{\prime}$. $24 \mathrm{D}$, male
Male treatment room, $133^{\prime} \times 10^{\prime}$,

treatment room irrigation cubicles. $24 \mathrm{E}$, treatment room lavatory

Male waiting room. $25 \mathrm{~A}$, male waiting room lavatory. $25 \mathrm{~B}$, entrance to male waiting room

Male patients' main entrance

Store room, $6^{\prime} \times 10^{\prime} \times 10^{\prime}$

Staff common room $10^{\prime} \times 10^{\prime} \times 10^{\prime}$

Staff cloakroom and lavatories, $10^{\prime} \times 5^{\prime} \times 10^{\prime}$

Staff entrance

Staff entrance
Small staff kitchen, $5^{\prime} \times 5^{\prime} \times 10^{\prime}$

Dispensary, room 17.-Working bench fitted with gas taps, Bunsen burner and gas or electric water heater, desk, filing cabinet, poison cupboard, shelves (glass), store cupboard, table, chairs, small refrigerator, hot and cold water lavatory basin.

Patients' waiting rooms, 12 and 25.-These should contain an adequate number of chairs, not benches, and one or two tables and wall electric radiators.

Clerks' office, room 6.-Two desks, four large filing cabinets, four small card indexes. Dividing partition at waiting-room end. Two desk chairs, four straight chairs, two desk lamps. Two electric wall radiators.

Medical officers' and staff common rooms, 3 and 28.-Desk, table, lounge chairs, straight chairs, and carpets.

Staff kitchen, room 31.-Small refrigerator, small electric or gas cooker, small kitchen sink, small kitchen dresser and table.

\section{V.D. in-patient department}

Beds should be available in each district and situated in some county institution adjacent to the clinic.

No. 1 central district clinic.-This should have the use of 24 beds in order to accommodate the ordinary V.D. district male, female and child patients and the resistant gonorrhoea cases which require special treatment, as well as patients with severe reactions after syphilis treatment.

Neurosyphilis department attached to the central district clinic.-Neurosyphilis cases should be in the same building as the V.D. bed cases. There should be six beds, two fully trained female nurses and facilities for treatment by malaria therapy and the Kettering hypertherm cabinet. This department would also be used when necessary for cases of resistant gonorrhoea.

No. 2 and No. 3 district clinics.-The clinics should have the use of 18 beds each-to be divided between male, female, and child patients-at some adjacent institution for ordinary V.D. in-patients. In cases of neurosyphilis and of persistently resistant gonorrhoea, the patients should be transferred to central clinic beds and to the neurosyphilis section.

Medical equipment.-In addition to that laid down by the Ministry of Health, the equipment should include the following electrical instruments for use in diagnosis : 1 urethroscope, 1 endocystoscope, 2 auriscopes, 2 ophthalmoscopes, 1 electric cautery with points of various sizes, 2 proctoscopes, 2 medium sized vaginal specula, 2 sinus transilluminators adapted for use with a light-main transformer.

\section{Laboratory service at central laboratory}

The following should be provided by this service.

(1) Serological tests for all three districts on two days each week. The Harrison-Wyler and Richardson modification Wassermann techniques should be employed as well as Kahn and Meinicke precipitation tests.

(2) Routine examination proceedings for cerebrospinal fluid including colloidal gold and colloidal mastic procedures. 
(3) Routine haematological investigations.

(4) Renal function procedures.

(5) Liver function procedures.

(6) Reliable cultural methods for gonococci, Ducrey's bacillus and pathogenic organisms affecting the genito-urinary system.

(7) Animal tests.

(8) Pathological investigations of placentas of doubtful cases and of women under treatment for syphilis at all county institutions.

(9) Dark-ground investigations of biopsy material from the clinic and the general practitioners' section of the service.

(10) Preparation and storage of culture media and transport media.

(11) Organization of transport of cultured material.

(12) Cooperation in clinicopathological research into fusospirochaetosis infections, Trichomonas vaginalis infections and monilial infections and their possible relation to resistant gonorrhoea and non-specific infections of the genito-urinary tract.

(13) Section cutting and diagnosis on biopsy material in lymphogranuloma venereum, granuloma inguinale and chancroid, and for differential diagnosis of late cutaneous syphilis.

(14) Section cutting and diagnosis of necropsy material.

(15) Routine post-mortem investigation of patients who die during or after completion of treatment for venereal diseases in the three districts.

(16) Preparation of sterile solutions such as may be required for intravenous use at district clinics and associated V.D. wards.

(17) Material and equipment, supplies and supervision at auxiliary clinic laboratories.

(18) Instruction of clinic male attendants and female nurses and laboratory staffs in the various elementary procedures of V.D. clinical pathology.

(19) Instruction of district V.D. medical officers and associated services in V.D. clinical pathology.

(20) Equipment necessary for all investigation procedures.

\section{Laboratory service at district clinic auxiliary laboratories}

Investigation facilities should be provided as follows.

(1) One microscope fitted for reliable dark-ground investigation, permanently set up, with adequate illumination for immediate use at any time.

(2) One microscope fitted for investigations by oil-immersion $1 / 12$ th objective and by high and lower power objectives as required.

(3) Thin slides and cover-slips for dark-ground investigation.

(4) Slides and cover-slips for smear and other investigations.

(5) Platinum loops.

(6) One still for the rapid preparation of triple distilled water.

(7) Sterile fluids of various kinds for intravenous and other clinical uses.

(8) Stains as required.

(9) Culture media for transporting culture material and for culture at laboratories.

(10) Glass pipettes for taking culture and investigation material.

(11) Capillary tubes for serum material.

(12) Blood tubes for serological material.

(13) Special glass tubes for cerebrospinal fluid.

(14) Containers and formol saline for biopsy material.

(15) Containers for urine specimens.

(16) Materials for qualitative urine investigations.

\section{Dispensary arrangements}

These will be controlled by the service dispenser from the central dispensary at No. 1 central clinic who will be directed by the specialist V.D. officer. The following will be provided.

(1) One chief dispenser, stationed at No. 1 central clinic, who will also preside over the dispensary at one general clinic and one combined congenital and antenatal clinic at each district clinic each week.

(2) One part-time dispenser at each district clinic working under the direction of the service dispenser.

(3) Storage of service drugs at the central district dispensary and supplies at No. 2 and No. 3 district clinics.

(4) Purchase, supply, repairs and replacement of service clinical instruments and appliances.

(5) Preparation of mixtures and solutions required by the clinic and V.D. wards.

(6) Clerical work of dispensing service, which will be performed by the service dispenser and his part-time dispensers, and will include clinic day books, poison registers, returns to clinic service chief clerk and ordering of drugs from wholesale chemists.

(7) Transport of drugs and return of empty containers to wholesale druggists.

(8) Supply of drugs required for V.D. treatment to general practitioners employed by the service in each district from stores which will be maintained at each district clinic. 


\section{Clerical services}

These will provide three district clinic male clerks under the control of the chief clerk of the clinical section and three female clinic clerks under the control of the almoner's chief clerk and under the joint direction of the specialist V.D. officer and the assistant lady almoner. The clerks will be responsible for the following services.

(1) Alphabetical name and address card index system and numerical card index system

kept for all patients attending the clinics and treated in the V.D. wards.

(2) Records of pathological material sent to the central laboratory and reports returned

from the laboratory.

(3) Entry of laboratory reports on the clinical cards in use at the clinic.

(4) Filing of records of patients under, on rest from and discharged from treatment.

(5) Weekly statistical returns to the central laboratory in accordance with the requirements of the Ministry of Health.

(6) Weekly statistical returns to the almoner's department of defaulters from treatment and contact information.

(7) Weekly statistical returns to the clinical section central office in accordance with the requirements of the annual report to the Ministry of Health.

(8) Filing of answered and unanswered letters received at the district clinic.

(9) Typing in triplicate all letters sent from the clinic and filing one copy at the clinic and returning one copy to chief clerks of the various sections.

(10) Maintaining stationery supplies at the district clinic.

(11) Keeping postage accounts and making weekly postal returns.

(12) Posting of letters and pathological specimens.

(13) Care, disposal and return of medical books and periodicals in the district clinic library which have been lent to medical officers and to the medical clinic staff.

\section{Scabies associated service}

Scabies infection is so often associated with venereal disease and the genital scabies burrow is so often the site of a chancre of primary syphilis, which develops when the scabies infestation is apparently cured, that one is justified in regarding scabies as the seventh venereal disease. Again, many people acquire scabies from sexual intercourse. Such cases should be kept under observation and blood tests should be made for at least three months after the appearance of the scabies rash.

In consideration of the above, it would be a great advantage to the venereal diseases service if one of the disinfestation centres of each district could be situated adjacent to the district V.D. clinic and could work under the supervision of the district clinic medical officers. The centre could make use of the clinic sterilizing plant for the disinfestation of the clothing of patients. It should be constructed so that male and female patients could be treated concurrently in separate sections of the same building. One waiting room, one treatment room, three bathrooms, and one drying room would be required in both the male and female sections of such a centre.

\section{Associated consultant service}

The following consultants should be available to attend each district clinic in consultation with the medical officers of the clinic at regular convenient intervals on a sessional basis : surgeon dentist ; ear, nose and throat specialist ; urologist ; orthopaedic surgeon ; children's specialist ; ophthalmologist ; gynaecologist.

A consultant general physician with special skill in neurology should be available at the neurosyphilis section attached to the No. 1 central clinic for consultations on neurosyphilis cases and for the preliminary general examination of patients selected for treatment by means of the hypertherm cabinet.

Facilities for radiological investigations on selected cases should also be available, preferably at the institution which accommodates the V.D. beds in each district. 